\title{
Ensemble Clustering based on Heterogeneous Dimensionality Reduction Methods and Context-dependent Similarity Measures
}

\author{
Augustine S. Nsang ${ }^{1}$, Irene Diaz ${ }^{2}$ and Anca Ralescu ${ }^{3}$ \\ ${ }^{1}$ CS Department, School of Inf. Tech. and Computing, American University of Nigeria \\ Yola By-Pass, PMB 2250, Yola, Nigeria \\ ${ }^{2}$ Department of Computer Science, University of Oviedo, Spain \\ ${ }^{3}$ EECS Department, University of Cincinnati, Cincinnati, OH 45221-0030, USA \\ augustine.nsang@aun.edu.ng, sirene@uniovi.es,anca.ralescu@uc.edu
}

\begin{abstract}
This paper discusses one method of clustering a high dimensional dataset using dimensionality reduction and context dependency measures (CDM). First, the dataset is partitioned into a predefined number of clusters using CDM. Then, context dependency measures are combined with several dimensionality reduction techniques and for each choice the data set is clustered again. The results are combined by the cluster ensemble approach. Finally, the Rand index is used to compute the extent to which the clustering of the original dataset (by CDM alone) is preserved by the cluster ensemble approach.
\end{abstract}

Keywords: dimensionality reduction, context dependency measures, rand index, cluster ensemble approach

\section{Introduction}

Given a collection of $\mathrm{n}$ data points (vectors) in high dimensional space, it is often helpful to be able to project it into a lower dimensional space without suffering great distortion [1]. In other words, it is helpful to embed a set of $m$ points in $n$-dimensional space into $r$ dimensional space, where $\mathrm{r} \ll \mathrm{n}$. This operation is commonly known as dimensionality reduction.

There are many known methods of dimensionality reduction. In some of these methods, each attribute in the reduced set is a linear combination of the attributes in the original data set. Such methods include Random Projection (RP), Singular Value Decomposition (SVD), Principal Component Analysis (PCA), Kernel Principal Component Analysis (KPCA), Discrete Cosine Transform (DCT) and Latent Semantic Analysis (LSA) [5]. Therefore, in effect, to compute a data point in the new dimensions one needs to actually know all of the original dimensions.

Other dimensionality reduction methods, however, reduce a dataset to a subset of the original attribute set [6]. These include those studied in [16], namely, the Direct Approach (DA), the Combined Approach (CA), the Variance Approach (VAR), the Top-Down Approach (TD), the Bottom-Up Approach (BU), the Weighted Attribute Frequency Approach (WAF) and the Best Clustering Performance Approach (BCP) which will be illustrated further in this paper. Other filtering approaches for high dimensional data can be found in [9] [11], [12], [2].

An application of dimensionality reduction is the clustering of high dimensional data. A common view of clustering is as the assignment of a data set into subsets (called clusters) so 
that data in the same cluster are similar in some sense [4], [8], [17] and data assigned to different clusters are as dissimilar as possible. Clustering is a method of unsupervised learning, and much used for data analysis in many fields, including machine learning, data mining, pattern recognition, image analysis and bioinformatics. This paper discusses a method of partitioning an $n$-dimensional data set $\mathrm{D}$ containing $m$ data points, into k clusters, $\mathrm{k} \ll \mathrm{n}$, based on integrating context dependency measures and dimensionality reduction techniques, such that the clusters in the original dimensions are preserved as much as possible by the clusters in the reduced dimensions. From this point on, the paper is organized as follows. Section 2 describes the concepts of context-based divergence and context-based similarity. Sections 3 and 4 illustrate the use of these concepts in connection with contextbased cluster structure, the computation of the similarity and divergence between a data sample and a cluster and between two clusters in a context-based environment. Section 5 reviews the dimensionality reduction approaches used in this study and in Section 6 these are used in conjunction with the context dependency measures in a cluster ensemble approach. Section 7 presents illustrative experimental results on a small artificial data set. Section 8 provides brief concluding remarks.

\section{Context-Based Proximity Measurements [19]}

The distance between two data vectors, as measured by various distance formulae is static. Once computed, it does not change as long as the vectors do not change. Moreover, there could be cases where the vectors change but the distance between them remains constant. This is possible when the two vectors are translated by a fixed amount in the same direction in multidimensional space. But the perception of distance is not static. For example, consider the problem of measuring the proximity between two geographical locations, say two cities, for example Cincinnati, Ohio and Indianapolis, Indiana, based only on their geographical information, and not taking into consideration any other locality. Since the locations of these cities are different, each of these two cities perceives the other as most different. Now consider adding a third location, for example, Los Angeles, California. Now, the perceived proximity between Cincinnati and Indianapolis is modified - has become larger, since Los Angeles is much farther than any of these two cities, and by comparison Cincinnati and Indianapolis seem to be almost the same. Similarly, if now another city, say, Lagos Nigeria is added, proximity between the three American cities is further increased. This modification of proximity perception is evident when people discuss about distances. It is not uncommon for someone from Lagos to feel, for example that if one traveled to Cincinnati, one has also almost "arrived" in Los Angeles. Yet, once in Cincinnati this feeling is not present anymore, since in the context of distances in the US, Cincinnati and Los Angeles are pretty far. This example illustrates the effect of the context in perception of proximity. If proximity perception is to be measured in the interval $[0,1]$, then in the first case described above, the proximity between Cincinnati and Indianapolis is almost 0 . As other, more distant cities are added, it increases almost to 1 (almost as if Cincinnati and Indianapolis were co-located). The context-based divergence is an approach (proposed in [19] and further explored in [20,21]), which captures and explores this notion of perceptual proximity and it will be reviewed in this paper. 


\section{Terminology}

Unless otherwise specified, the following terminology will be used in this paper:

- $X=X_{i, e}, i=1, \ldots, m ; e=1 \ldots, n$ denotes a data matrix each with $m$ data vectors, each with $n$ attributes or dimensions. $X_{i, e}$ can take any real value consistent with its semantics. $X_{i, *}$ denotes the $i^{\text {th }}$ data sample (row), and $X_{*, e}$ denotes the column corresponding to the $e^{\text {th }}$ variable.

- $\quad \mathrm{C}_{m}^{l}$ denotes a cluster identified by label 1 , consisting of $m$ data samples (cluster members). That is, $\left|\mathrm{C}_{m}^{l}\right|=m$.

- $\quad \mathrm{C}^{l}$ denotes a cluster of unspecified size identified as $l$, and $i \in \mathrm{C}^{l}$ refers to the $i^{\text {th }}$ member in $\mathrm{C}^{l}$.

- $\mathrm{C}_{m}$ stands for an unlabeled cluster of size $m$, such that $i \in \mathrm{C}_{m}$ refers to the $i^{\text {th }}$ element in it.

Definition 1. [19] The vertical context ( $v$-context) for a sample data vector is the collection of the remaining data vectors. Generalizing, the $v$-context for a collection of data vectors is the intersection of the $v$-contexts of the individual vectors. Mathematically:

$$
\begin{aligned}
& v-\text { context }_{X}\left(X_{i}, *\right)=\left\{x \in X: x \neq X_{i, *}\right\} \\
& v-\text { context }_{X}(N \subset X)=\cap_{x \in N} v-\text { context }_{X}(x)
\end{aligned}
$$

In the example used earlier, while measuring the proximity between two cities, the vertical context consists of all the other cities in the data set. Similarly, the horizontal context of a sample attribute is the collection of the remaining attributes.

Definition 2. [19] The context dependent divergence between data vectors $X_{i, *}$ and $X_{j, *}$ with respect to attribute e is defined by:

$$
D_{e}(i, j)=\frac{\left[X_{i, e}-X_{j, e}\right]^{2}}{\delta_{X}^{2}(i, e)}
$$

where $\delta_{X}^{2}(i, e)$, the variance of $X_{i, e}$ about the $i^{\text {th }}$ point along the $e^{\text {th }}$ dimension, is defined by:

$$
\delta_{X}^{2}(i, e)=\sum_{k=1}^{m}\left(X_{i, e}-X_{k, e}\right)^{2}
$$


Generalizing over all the attributes, the overall context dependent divergence between data vectors $X_{i, *}$ and $X_{j, *}$ is defined as the arithmetic mean of their divergences over all the attributes. That is:

$$
D(i, j)=\frac{1}{n} \sum_{e=1}^{n} D_{e}(i, j)
$$

Finally, the similarity (or proximity) between two data vectors $i$ and $j$ is given by:

$$
S(i, j)=1-D(i, j)
$$

To illustrate how the above definitions capture the effect of context in evaluating the proximity between two data points, consider the data set $X_{\delta}$ given by $3 \times 2$ matrix in the equation below:

$$
X_{\delta}=\left[\begin{array}{cc}
1 & 4 \\
2+\delta & 3+\delta \\
50 & 100
\end{array}\right]
$$

In this example, $X_{\delta_{(2, *)}}$, the second row, is the $v$-context for the rows one and three. The context divergence between the 1st and 3rd row of this matrix, as a function of $\delta$ is computed to be

$$
D_{\delta}(1,3)=\frac{1}{2}\left(\frac{49^{2}}{(1+\delta)^{2}+49^{2}}+\frac{96^{2}}{(1-\delta)^{2}+96^{2}}\right)
$$

\begin{tabular}{|c|c|c|c|c|}
\hline$\delta$ & 0 & 100 & $\ldots$ & 1000 \\
\hline & {$\left[\begin{array}{ll}1 & 4\end{array}\right]$} & {$[1$} & & {$[1$} \\
\hline$X_{\delta}$ & 23 & 102103 & $\ldots$ & 10021003 \\
\hline & {$\left[\begin{array}{ll}50 & 100\end{array}\right]$} & {$\left[\begin{array}{ll}50 & 100\end{array}\right]$} & & 100 \\
\hline$D_{\delta}(1,3)$ & 0.9997 & 0.3376 & $\ldots$ & 0.0058 \\
\hline$S_{\delta}(1,3)$ & 0.0003 & 0.6624 & $\ldots$ & 0.9942 \\
\hline
\end{tabular}

Obviously, $\lim _{\delta \rightarrow \infty} D_{\delta}(1,3)=0$ and hence, $\lim _{\delta \rightarrow \infty} S_{\delta}(1,3)=1-\lim _{\delta \rightarrow \infty} D_{\delta}(1,3)=1$.

The evolution of the values of $X, D_{\delta}(1,3)$ and $S(1,3)$ when $\delta=0,100,200, \ldots, 1000$ is shown in the table below.

Figure 1 illustrates the perceptual proximity for the data in this example. 

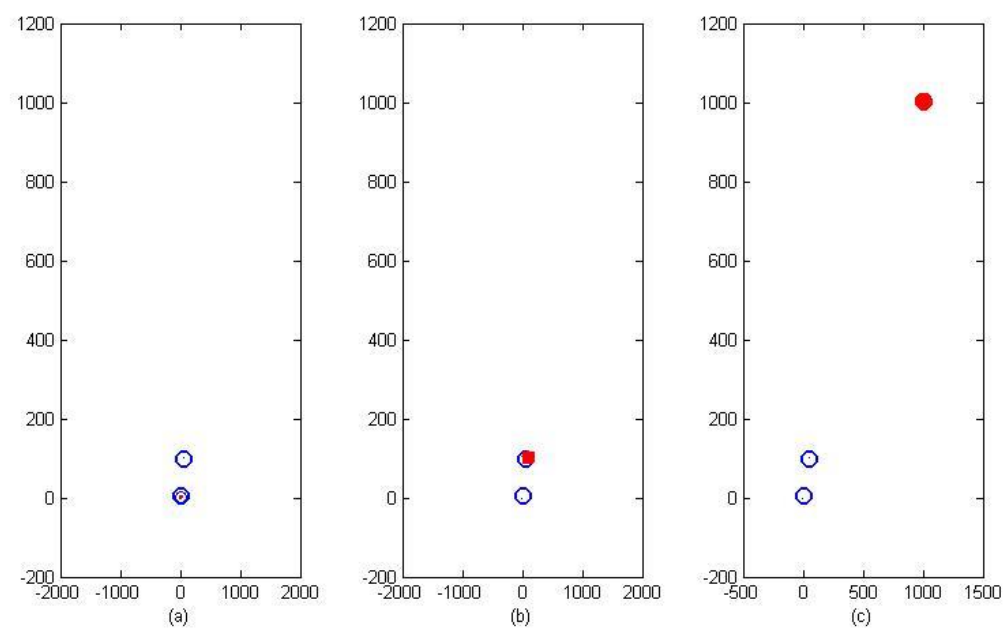

\section{Figure 1. Illustration of the change in perception of proximity for the data used in the above example.}

The values of $\delta$ are 0 , in (a), 100 in (b) and 1000 in (c). In each figure the blue circles show the rows one and three of $X_{\delta}$, while the red dots (very small in (a)) correspond to the changing positions of the second row as $\delta$ changes.

\section{Context-dependent Cluster Structure}

Taking into account context the notion of context dependent clustering can be defined (see [19]). Although a common approach used in pattern recognition, clustering remains a somewhat ill-defined problem: the objective is to find good groupings/clusters of data point with little or no information on the ground truth. A discussion of the issues underlying cluster validity can be found in [17]. The set of data vectors that satisfy certain criteria of proximity are grouped into clusters to reduce information load and facilitate information processing and interpretation.

Traditional approaches to clustering consider a cluster as a lump of data that is characterized by measures such as within cluster distance and between cluster distance [7][3] which measure respectively, the cluster tightness and separation. But these approaches are based on the assumption that all cluster members are equally significant from the point of view of measuring cluster properties and making decisions about cluster expansion [5][8]. As pointed out in [19], in real life, there are many situations in which assumptions of "equal significance" of all cluster members do not hold. If the clusters are represented as "clouds" in a multi-dimensional space, then the members near the center of a cluster may be considered more representative of the cluster properties. From the point of view of deriving cluster properties, the cluster members belonging to different regions of the clusters must be viewed differently. There should be a way to differentiate between the cluster members based on their significance to the cluster they belong to. One way to achieve this is by attaching weights to the cluster members.

In the spirit of using context to determine the significance of each member and not rely on static schemes of weighing cluster members, this section discusses a method of cluster representation which assigns weights to cluster members based on their context dependent divergence with other members in the cluster [19]. The idea is to define the significance of a 
given cluster member in a way which reflects its context dependent similarity with other cluster members.

Definition 3. (Perfect Cluster [19]) When all members of a cluster have identical attribute values, the cluster is called a perfect cluster. More precisely, a perfect cluster is a set of $m$ vectors $v_{i}, i=1, \ldots, m$ of dimension $n$ (attributes of a data item), such that:

$$
v_{i, k}=a_{k}, i=1, \ldots, m ; k=1, \ldots, n
$$

Thus, in fact, the perfect cluster is trivial - a collection of identical data points.

Definition 4. (Weight of a cluster member [19]) The weight, $\rho_{k}$, of the member $k \in C_{m}$, is defined as the normalized similarity of $k$ with all the remaining members. More precisely:

$$
\rho_{k}=\left\{\begin{array}{cl}
1 & \text { if } \mathrm{m}=1 \\
\frac{\sum_{l=1, l \neq k}^{m} S(l, k)}{\sum_{j=1}^{m} \sum_{l=1, l \neq j}^{m} S(l, j)} & \text { otherwise }
\end{array}\right.
$$

It is easy to see that

$$
\sum_{k=1}^{m} \rho_{k}=1
$$

For example, when the cluster contains only two elements, i.e. becomes the above formula yields respectively:

$$
\rho_{1}=\frac{S(2,1)}{S(2,1)+S(1,2)} ; \quad \rho_{2}=\frac{S(1,2)}{S(2,1)+S(1,2)}
$$

Therefore, the weights $\rho_{k}$ define a convex combination. Moreover, if $S(i, j)=S(j, i)$, for all pairs of clusters elements $i, j \in C_{m}$, when $\rho_{k}=\frac{1}{m}$. In particular this is true for the perfect cluster.

\section{Similarity Within and Between Clusters 0}

To measure the tightness of and separation between clusters, a similarity measure must be defined. A context dependent similarity measure between a data item and a cluster, on one hand, and two clusters, on the other hand, can be defined based on the context dependent divergence and similarity measures defined above.

\subsection{Similarity between a data item and a cluster}

The similarity between a data item $v$ and a cluster $C_{m}, S\left(v, C_{m}\right)$, is defined as convex aggregation of the similarity between $v$ and the cluster members $i$, determined by the weights $\rho_{i}$. 
That is,

$$
S\left(v, C_{m}\right)=\sum_{i=1}^{m} \rho_{i} S(v, i)
$$

Similarly, to account for the asymmetry, $S\left(C_{m}, v\right)$ can be defined as

$$
S\left(C_{m}, v\right)=\sum_{i=1}^{m} \rho_{i} S(i, v)
$$

\subsection{Similarity between clusters}

Similarly to data item to cluster similarity, the cluster to cluster similarity is defined as a convex combination of similarities. Let $C$ and $C^{\prime}$ denote two clusters.

Then $S\left(C, C^{\prime}\right)$ is defined as

$$
S\left(C, C^{\prime}\right)=\sum_{i \in C} \rho_{i} S\left(i, C^{\prime}\right)
$$

which can be further written as

$$
S\left(C, C^{\prime}\right)=\sum_{i \in C} \rho_{i} \sum_{j \in C^{\prime}} \rho_{j} S(i, j)
$$

From the asymmetry of the item-to-item similarity, it follows that

$$
S\left(C, C^{\prime}\right) \neq S\left(C^{\prime}, C\right)
$$

Remark 1. In the above, for ease of notation, the same symbol, $S$, was used to denote three different similarity measures, item-to-item similarity, item-to-cluster similarity, and clusterto-cluster similarity although these measures of similarities are defined between different structures.

The context dependent measures (CDMs) of similarity are used in an agglomerative clustering procedure in the original high dimensional data set, and also in conjunction with several methods of dimensionality reduction. To this end, the CDMs are symmetrized, as follows:

$$
S_{s y m}(x, y)=\frac{S(x, y)+S(y, x)}{2}
$$

where $S(x, y)$ denotes the item-to-item similarity.

\section{Dimensionality Reduction Approaches}

The basic description of the dimensionality reduction problem used in this paper is as follows: Given a data set represented as an $m \times n$ matrix $D$, find a representation of $D$ as an $m \times r$ matrix $D_{r}, r<n$ (usually much smaller than $n$ ) subject to some constraints (or optimization criterion). Some of the dimensionality reduction techniques studied in [14 - 16] and used subsequently in this paper are briefly described below.

\subsection{The Variance Approach (VAR)}

The Variance approach starts with an empty set, $I$ of dimensions and adds dimensions of $D$ to this set in decreasing order of their variances [15]. That means that a set $I_{r}$ with $r$ dimensions contains the dimensions of top $r$ variances. This approach assumes that the 
discriminatory power of a dimension depends directly on the variance of the data along that dimension; dimensions of low variance are left out as they would fail to discriminate between the data. Indeed, in an extreme case where all the values along a dimension are equal, and therefore, the variance is 0 , it is impossible to distinguish between data points based on that dimension alone. Thus, letting $I_{r}=\left\{i_{1}, \ldots, i_{r}\right\} \subset\{1, \ldots, n\}$ be the collection of dimensions corresponding to the top $r$ variances, the reduced database, $D_{r}$ is obtained by extracting the data corresponding to the selected dimensions. That is, $D_{r}=D\left(:, I_{r}\right)$. Given that $D$ is an $m x$ $n$ data set, $D_{r}$ is an $m \times r$ data set whose $i^{\text {th }}$ column is the column of the original database of $i^{\text {th }}$ largest variance.

\subsection{The Combined Approach $(C A)$}

The Combined Approach selects the combination of $r$ attributes which best preserve the inter-point distances, and reduces the dataset to a dataset containing only those $r$ attributes. This approach consists of the following steps:

1. Determine the extent to which each attribute preserves the inter-point distances. In other words, for each dimension $i \in\{1, \ldots, n\}$ compute

$$
\begin{aligned}
& m_{i}=\min _{u, v}\left\{\frac{\left\|f_{i}(u)-f_{i}(v)\right\|^{2}}{\|u-v\|^{2}}\right\} \\
& M_{i}=\max _{u, v}\left\{\frac{\left\|f_{i}(u)-f_{i}(v)\right\|^{2}}{\|u-v\|^{2}}\right\}
\end{aligned}
$$

where $u$ and $v$ are rows of $D, f_{i}(u)$ and $f_{i}(v)$ are the corresponding rows in the dataset reduced to the single attribute $i$.

2. Compute the average distance preservation, mid $_{i}$, for each attribute $i$ :

$$
\operatorname{mid}_{i}=\frac{m_{i}+M_{i}}{2}
$$

3. Reduce $D$ to $D_{r}$ as follows: Let $I_{r} \subset\{1, \ldots, n\}$ be defined as $I_{r}=\left\{i_{1}, \ldots, i_{r}\right\}$, where $i_{1}, \ldots, i_{r}$ is the collection of the $r$ dimensions of $D$ whose average mid $_{i}$ value is maximum. In other words,

$$
\mathrm{I}_{\mathrm{r}}=\operatorname{argmax}_{\mathrm{C}}\left(\frac{1}{|r|} \sum_{i \in C} \operatorname{mid}_{i}\right)
$$

Then $\mathrm{D}_{\mathrm{r}}$ is defined as $\mathrm{D}_{\mathrm{r}}=\mathrm{D}\left(:, \mathrm{I}_{\mathrm{r}}\right)$.

\subsection{The Direct Approach $(D A)$}

Like for the Combined Approach, to reduce a dataset $D_{m \times n}$ to a dataset containing $r<n$ columns, the Direct Approach selects the combination of $r$ attributes which best preserve the 
inter-point distances, and reduces the original dataset to a dataset containing only those $r$ attributes. However this done in a different way according to the following steps:

1. Generate all possible combinations of $r$ attributes from the original $n$ attributes.

2. For each combination, $C$, generated at the previous step, compute $m_{C}$ and $M_{C}$ as follows:

$$
\begin{gathered}
m_{C}=\min _{u, v} \frac{\left\|f_{C}(u)-f_{C}(v)\right\|^{2}}{\|u-v\|^{2}} \\
M_{C}=\max _{u, v} \frac{\left\|f_{C}(u)-f_{C}(v)\right\|^{2}}{\|u-v\|^{2}}
\end{gathered}
$$

where $u$ and $v$ are rows of $D$, and $f_{C}(u)$ and $f_{C}(v)$ are the corresponding rows in the dataset reduced to the attributes in $C$. Compute the average distance preservation for this combination of attributes as

$$
\operatorname{mid}_{C}=\frac{m_{C}+M_{C}}{2}
$$

Select the subset of dimensions $\mathrm{C}_{0}$ as

$$
\mathrm{C}_{0}=\operatorname{argmin}_{\mathrm{C}}\left|\operatorname{mid}_{\mathrm{C}}-1\right|
$$

Thus $D_{r}$ is given as $\mathrm{D}_{\mathrm{r}}=\mathrm{D}\left(:, \mathrm{C}_{\mathrm{o}}\right)$.

The difference between the Combined and Direct approaches is that for the Combined Approach, to reduce a data set $D$ containing $n$ attributes to a data set $D_{r}$ containing $r$ attributes, the average distance preservation is first calculated for each attribute, and all combinations of $r$ attributes (from the $n$ attributes of D) are then generated. $D_{r}$ then becomes the dataset containing the $r$ attributes of $D$ whose average mid $_{i}$ value is maximum. With the Direct Approach, on the other hand, to reduce a data set $D$ containing $n$ attributes to a data set $D_{r}$ containing $r$ attributes, we first generate all combinations of $r$ attributes from the $n$ attributes of $D$. To find the average distance preservation for any combination of attributes, $C$, we reduce the original dataset directly to the dataset containing only the attributes in $C$, and then compute the average distance preservation for this combination using the formulas above. $D_{r}$ then becomes the dataset containing the $r$ attributes whose average distance preservation is maximum.

\subsection{Top-down Approach (TD)}

The Top-Down dimensionality reduction approach considers subsets of attributes reduced by one attribute at a time. To reduce a data set $D$ containing $n$ attributes to a data set $D_{r}$ containing $r$ attributes, the Top-Down Approach starts with the original $n$ attributes of $D$, and reduces it successively to the subset of $n-i, i=1,2, \ldots$ attributes, such that a certain criterion is satisfied, until $n-i=r$. For example, the criterion could be to preserve as well as possible $k$-means clustering [14-16]. 


\subsection{The Bottom-up Approach $(B U)$}

Contrary to the Top-Down dimensionality reduction approach, the Bottom-Up approach considers subsets of attributes increased by one attribute at a time. Starting with the subset $S_{1}$ containing the single attribute, $i_{1}$, of $D$, which best preserves $k$-means clustering, it increases it to the subset $S_{2}$ of two attributes $\left(i_{1}, i_{2}\right)$ which best preserve $k$-means clustering. The process continues until the subset $S_{r}$ of $r$ attributes $\left(i_{1}, \ldots, i_{r}\right)$ which best preserve $k$ means clustering has been obtained.

Remark 2. It should be noted that each of these approaches implements a heuristic and that the final subset of attributes obtained is not guaranteed to be optimal with respect to the criterion selected. However, in experiments, these approaches were found to be more effective.

\subsection{The Weighted Attribute Frequency Approach (WAF)}

In each of the approaches considered above, attributes/dimensions were considered implicitly equally important. On the other hand, from applying each of these approaches, and inspecting the corresponding selected attribute subsets it can often be seen (depending on the actual data set $D$ ) that some attributes appear more often than others. The weighted attribute frequency approach (WAF) aims at taking this into consideration. To reduce an $m \times n$ data set $D$ to an $m \times r$ data set $D_{r}$, with $r<n$ WAF works as follows:

1. Generate $D A(r), C A(r), \operatorname{Var}(r), T D(r)$, and $B U(r)$ each of which is the collection of attributes obtained when the given dimensionality reduction method is applied to reduce it to $r$ attributes.

2. Assign weights to an attribute $i$ according to its selection by each of these methods. From experimental studies, the weights were assigned as

$$
w(i)=\left\{\begin{array}{c}
3 \text { if } i \in\{N T D(r), N B U(r)\} \\
1 \text { if } i \in\{D A(r), C A(r), \operatorname{Var}(r)\}
\end{array}\right.
$$

Thus WAF( $r$ ) becomes the collection of the $r$ attributes of $D$ attributes of largest weight.

\subsection{The Best Clustering Performance Approach $(B C P)$}

A consequence of distance preservation is that when used in algorithms where distance is computed, the original or reduced data should yield the same results in all algorithms based on inter-point distance. Such algorithms include classification and clustering. Given $k$, and the set $\mathrm{DR}=\{\mathrm{CA}(\mathrm{r}), \mathrm{DA}(\mathrm{r}), \operatorname{Var}(\mathrm{r}), \mathrm{TD}(\mathrm{r}), \mathrm{BU}(\mathrm{r})\}$ whose elements are defined as in Section 5.6 above, $B C P(k, r)$ is the element of $D R$ which best preserves $k$-means clustering with a subset of $r$ attributes. For example, if the Var approach best preserves k-means clustering when $D$ is reduced to five attributes, for $k=3$, then $B C P(3,5)=\operatorname{Var}(5)$. On the other hand, if the Top-Down Approach best preserves k-means clustering when $D$ is reduced to three attributes, for $k=3$, then $B C P(3,3)=T D(3)$. 


\section{Clustering in a High Dimensional Space based on Clustering in Reduced Dimensions}

The context dependent procedure (CDM) defined here is an agglomerative clustering algorithm which starts with clusters corresponding to each data item. Clusters are merged based on their context dependent similarity until the desired number of clusters is obtained. Algorithm 1 shows the exact description of the CDM based clustering procedure.

Algorithm 1: Cluster formation using the context dependent similarity measure

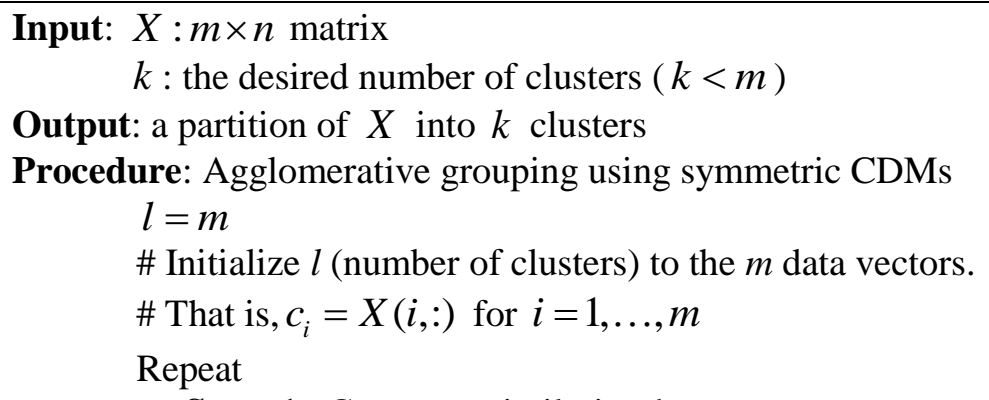

Output: a partition of $X$ into $k$ clusters

Procedure: Agglomerative grouping using symmetric CDMs

$$
l=m
$$$$
\text { \# Initialize } l \text { (number of clusters) to the } m \text { data vectors. }
$$$$
\text { \# That is, } c_{i}=X(i,:) \text { for } i=1, \ldots, m
$$

Repeat

Step 1. Compute similarity between every two clusters using the symmetric CDMs

Step 2. Find the most similar pair of clusters $c_{i}$ and $c_{j}$ in $X$

Step 3. Merge $c_{i}$ and $c_{j}$ and decrement $l$ by 1

Until $l \leq k$

Return all nonempty clusters

Let $D R=\{C A, D A$, Var, $T D, B U, W A F, B C P, P C A\}$. For each dimensionality reduction technique $\mathrm{C} \in D R$, clustering is obtained based on CDMs agglomerative clustering Algorithm 1. Then the ensemble clustering technique [4] is used to aggregate the multiple clustering results. The approach used in the current ensemble approach is inspired from and similar to that in [4], with the difference that in [4] all the clusters were represented by the same probabilistic model, determined by a parameter $\theta$ as a mixture of Gaussians.

For a data point $x_{i}, P(l \mid i, \mathrm{C})$, denotes the probability that $x_{i}$ belongs to cluster $l$, $l=1, \ldots, k$ under the dimensionality reduction $\mathrm{C}$. This probability is defined as

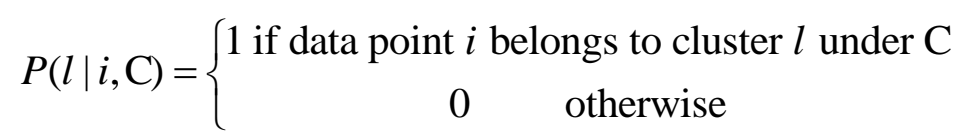

Next, assuming independence, the probability that the data points $x_{i}$ and $x_{j}$ belong to cluster $l$ under $\mathrm{C}$ is

$$
P(l \mid i, j, \mathrm{C})=P(l \mid i, \mathrm{C}) \times P(l \mid j, \mathrm{C})
$$

the probability that data points $x_{i}$ and $x_{j}$ belong to the same cluster under $\mathrm{C}$ is

$$
P(i, j \mid \mathrm{C})=\sum_{l=1}^{k} P(l \mid i, j, \mathrm{C})
$$


and finally, the probability that they belong to the same cluster is the average over clustering under all of the dimensionality reduction methods in $D R$ is

$$
P(i, j)=\frac{1}{|D R|} \sum_{\mathrm{C} \in D R} P(i, j \mid \mathrm{C})
$$

Note that in fact, from the definition of $P(l \mid i, \mathrm{C})$ it follows that $P(i, j)$ has the following properties:

1. $0 \leq P(i, j) \leq 1$,

2. $P(i, j)=0$ if the data points $x_{i}$ and $x_{j}$ never belong to the same cluster when CDM clustering is run with any of the dimensionality reduction methods in $D R$

3. $P(i, j)=1$ if the data points $x_{i}$ and $x_{j}$ belong to the same cluster when CDM clustering is run with every dimensionality reduction method in $D R$.

As in [4], the matrix $P(i, j), i, j=1, \ldots, m$ is used to define a conservative cluster similarity measure

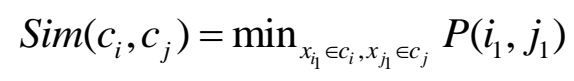

where $c_{i}$ and $c_{j}$ are clusters, which is used in an agglomerative clustering procedure identical to that Algorithm 1 in which Step 1 computes the similarity matrix $P$, and Step 2 evaluates $\operatorname{Sim}\left(c_{i}, c_{j}\right)$.

\section{Outliers}

Outliers are defined here as those points for which maximum similarity to any other data point is very small. These data points are initially discarded in the process of cluster formation. Approximately $10 \%$ of data points are removed this way in the current study. Then these points are assigned to the most similar of the final clusters obtained.

\section{Illustrative Experimental Results}

The approach described in the preceding sections is applied to a $70 \times 10$ data set shown in Table 1. The data set was clustered into 20 clusters in the original dimensions using Algorithm 1 and the context dependent measure of similarity. The clusters obtained are shown in Table 2. The data set was then reduced to seven dimensions, successively, using each of the eight dimensionality reduction techniques from the set $D R$ (in Section 6 above), and clustered using the context dependent measures of similarity for each reduction. Tables 3 -10 show the clusters obtained for each choice of dimensionality reduction technique. The similarity matrix $P$ is then computed and used to aggregate the clustering results in Tables 3 - 10. The final clusters obtained by the Cluster Ensemble Approach are shown in Table 11. 
Table 1: A small data se of 70 data points and 10 dimensiona.

\begin{tabular}{|c|c|c|c|c|c|c|c|c|c|}
\hline 75 & 0 & 190 & 80 & 91 & 193 & 371 & 174 & 121 & -16 \\
\hline 56 & 1 & 165 & 64 & 81 & 174 & 401 & 149 & 39 & 25 \\
\hline 54 & 7 & 172 & 95 & 138 & 163 & 386 & 185 & 102 & 96 \\
\hline 55 & 14 & 175 & 94 & 100 & 202 & 380 & 179 & 143 & 28 \\
\hline 82 & 21 & 197 & 87 & 88 & 181 & 360 & 177 & 103 & -9 \\
\hline 13 & 28 & 169 & 51 & 107 & 167 & 321 & 181 & 91 & 107 \\
\hline 40 & 8 & 160 & 52 & 77 & 129 & 377 & 133 & 77 & 77 \\
\hline 49 & 15 & 162 & 54 & 78 & 0 & 376 & 157 & 70 & 67 \\
\hline 44 & 35 & 168 & 56 & 84 & 118 & 354 & 160 & 63 & 61 \\
\hline 50 & 22 & 167 & 67 & 89 & 130 & 383 & 156 & 73 & 85 \\
\hline 62 & 42 & 170 & 72 & 102 & 135 & 408 & 163 & 83 & 72 \\
\hline 45 & 29 & 179 & 86 & 98 & 143 & 373 & 150 & 65 & 12 \\
\hline 61 & 36 & 186 & 58 & 85 & 155 & 382 & 170 & 81 & -24 \\
\hline 30 & 49 & 177 & 73 & 105 & 180 & 355 & 164 & 104 & 68 \\
\hline 51 & 43 & 174 & 88 & 112 & 158 & 399 & 184 & 94 & 46 \\
\hline 47 & 50 & 150 & 48 & 75 & 132 & 350 & 169 & 72 & 36 \\
\hline 68 & 56 & 171 & 59 & 82 & 145 & 347 & 176 & 61 & 84 \\
\hline 46 & 57 & 158 & 65 & 70 & 120 & 353 & 122 & 52 & 57 \\
\hline 73 & 63 & 193 & 63 & 119 & 154 & 392 & 175 & 90 & 73 \\
\hline 57 & 64 & 166 & 79 & 96 & 188 & 406 & 158 & 79 & -12 \\
\hline 28 & 71 & 181 & 93 & 83 & 251 & 390 & 189 & 183 & 50 \\
\hline 52 & 70 & 176 & 74 & 90 & 122 & 336 & 191 & 78 & 81 \\
\hline 36 & 78 & $i 53$ & 75 & 71 & 139 & 364 & 183 & 82 & 62 \\
\hline 64 & 85 & 200 & 66 & 103 & 157 & 413 & 143 & 92 & 4 \\
\hline 89 & 92 & 188 & 55 & 110 & 140 & 388 & 198 & 89 & 52 \\
\hline 58 & 77 & 183 & 101 & 109 & 128 & 389 & 195 & 60 & -34 \\
\hline 34 & 84 & 184 & 108 & 94 & 186 & 387 & 224 & 125 & 90 \\
\hline 31 & 99 & 195 & 61 & 95 & 161 & 407 & 168 & 97 & 10 \\
\hline 63 & 106 & 164 & 100 & 97 & 164 & 420 & 381 & 99 & -8 \\
\hline 65 & 113 & 202 & 83 & 117 & 147 & 400 & 301 & 96 & -37 \\
\hline 53 & 91 & 182 & 85 & 92 & 171 & 415 & 172 & 98 & -52 \\
\hline 72 & 120 & 163 & 68 & 99 & 136 & 339 & 152 & 76 & 13 \\
\hline 71 & 127 & 209 & 115 & 124 & 125 & 367 & 190 & 34 & 38 \\
\hline 59 & 134 & 155 & 70 & 106 & 137 & 368 & 148 & 111 & 9 \\
\hline 69 & 98 & 204 & 82 & 131 & 152 & 357 & 129 & 101 & 49 \\
\hline 79 & 141 & 216 & 45 & 69 & 178 & 378 & 137 & 80 & 69 \\
\hline 78 & 105 & 207 & 122 & 145 & 142 & 366 & 161 & 108 & 54 \\
\hline 35 & 148 & 178 & 129 & 113 & 200 & 385 & 188 & 74 & 48 \\
\hline 76 & 155 & 191 & 60 & 80 & 185 & 361 & 166 & 107 & -2 \\
\hline 66 & 112 & 189 & 136 & 101 & 170 & 422 & 159 & 106 & -57 \\
\hline 43 & 162 & 157 & 71 & 87 & 162 & 397 & 141 & 105 & 53 \\
\hline 96 & 169 & 223 & 89 & 79 & 168 & 427 & 167 & 115 & 74 \\
\hline 37 & 176 & 230 & 62 & 120 & 175 & 346 & 165 & 110 & 75 \\
\hline 41 & 183 & 214 & 96 & 126 & 159 & 404 & 144 & 71 & 59 \\
\hline 103 & 190 & 237 & 107 & 86 & 177 & 374 & 147 & 113 & -18 \\
\hline 110 & 197 & 156 & 69 & 73 & 166 & 434 & 138 & 120 & -11 \\
\hline 83 & 204 & 244 & 78 & 152 & 156 & 322 & 186 & 112 & 18 \\
\hline 86 & 211 & 221 & 57 & 108 & 144 & 369 & 171 & 119 & 30 \\
\hline 48 & 218 & 159 & 76 & 127 & 228 & 429 & 130 & 122 & 63 \\
\hline 90 & 225 & 173 & 103 & 115 & 149 & 379 & 205 & 118 & -14 \\
\hline 117 & 232 & 198 & 53 & 116 & 187 & 393 & 151 & 127 & 1 \\
\hline 42 & 239 & 205 & 143 & 104 & 184 & 359 & 197 & 87 & 34 \\
\hline 39 & 119 & 228 & 90 & 159 & 182 & 356 & 162 & 129 & 11 \\
\hline 24 & 246 & 212 & 81 & 134 & 192 & 370 & 142 & 68 & 64 \\
\hline 80 & 126 & 235 & 150 & 123 & 209 & 411 & 113 & 132 & 58 \\
\hline 93 & 253 & 251 & 77 & 133 & 191 & 441 & 202 & 88 & 37 \\
\hline 38 & 260 & 258 & 84 & 93 & 7 & 418 & 193 & 34 & 14 \\
\hline 70 & 133 & 196 & 114 & 111 & 173 & 448 & 182 & 86 & 4 \\
\hline 60 & 140 & 242 & 157 & 140 & 176 & 372 & 196 & 93 & 42 \\
\hline 32 & 267 & 265 & 121 & 118 & 150 & 414 & 209 & 54 & 88 \\
\hline 1 & 147 & 110 & 10 & 122 & 121 & 287 & 212 & 67 & 126 \\
\hline 77 & 274 & 272 & 128 & 141 & 189 & 324 & 155 & 75 & 40 \\
\hline 85 & 281 & 249 & 135 & 76 & 199 & 394 & 173 & 136 & 95 \\
\hline 27 & 288 & 211 & 102 & 166 & 196 & 381 & 216 & 85 & 66 \\
\hline 97 & 295 & 218 & 97 & 130 & 382 & 209 & 63 & 117 & 60 \\
\hline 100 & 302 & 225 & 142 & 129 & 117 & 363 & 219 & 100 & 56 \\
\hline 124 & 309 & 279 & 9 & 173 & 165 & 410 & 204 & 150 & 32 \\
\hline 26 & 316 & 286 & 149 & 148 & 206 & 455 & 200 & 95 & 80 \\
\hline 87 & 323 & 185 & 92 & 136 & 203 & 436 & 207 & 109 & 71 \\
\hline
\end{tabular}


International Journal of Advanced Science and Technology Vol.64 (2014)

Table 2: Clustering in the original space.

Table 3: Clustering under $V A R$

\begin{tabular}{|l||c||l|}
\hline \multicolumn{1}{|c||}{ Cluster Members } & Cluster D & \multicolumn{1}{|c|}{ Cluster Member's } \\
\hline \hline $1,5,39,45,50$ & $\mathbf{1}$ & $1,5,4,3,15$ \\
\hline $\begin{array}{l}2,3,11,12,13,15,20,24,25,26, \\
28,31\end{array}$ & $\mathbf{2}$ & $2,24,28,13,20,31,39,51$ \\
\hline $4,21,27$ & $\mathbf{3}$ & $6,17,14,22,23,7,10,11,19,25$ \\
\hline $6,14,43$ & $\mathbf{4}$ & $8,9,16,18,35,12,32,34$ \\
\hline $7,10,9,16,23,17,22,32,34$, & $\mathbf{5}$ & $21,27,38$ \\
$8,18,41$ & & \\
\hline $29,30,40,58$ & $\mathbf{6}$ & $26,50,33,37$ \\
\hline $33,37,35,47,48,53$ & 7 & 29,30 \\
\hline 36,42 & $\mathbf{8}$ & $36,43,54,41,42,44$ \\
\hline $38,52,44,54$ & $\mathbf{9}$ & 40,58 \\
\hline $46,51,49$ & $\mathbf{1 0}$ & $45,53,47,48$ \\
\hline 55,59 & $\mathbf{1 1}$ & 46,49 \\
\hline 56,69 & $\mathbf{1 2}$ & $52,64,66$ \\
\hline 57 & $\mathbf{1 3}$ & 55,59 \\
\hline $60,68,64$ & $\mathbf{1 4}$ & 56,67 \\
\hline 61 & $\mathbf{1 5}$ & 57 \\
\hline 62,66 & $\mathbf{1 6}$ & $60,63,68,69$ \\
\hline 63 & $\mathbf{1 7}$ & 61 \\
\hline 65 & $\mathbf{1 8}$ & 62 \\
\hline 67 & $\mathbf{1 9}$ & 65 \\
\hline 70 & $\mathbf{2 0}$ & 70 \\
\hline
\end{tabular}

Table 4: Clustering under $\mathrm{C} A$. Cluster Members

\begin{tabular}{|l|c|l|}
\hline \multicolumn{1}{|c|}{ Cluster Members } & Chuster D & \multicolumn{1}{|c|}{ Cluster Members } \\
\hline $1,5,53,34,39,47,48,45,50$ & $\mathbf{l}$ & $1,5,53,39$ \\
\hline $2,12,13,20,24,28$ & $\mathbf{2}$ & $\begin{array}{l}2,12,13,20,24,28,3,11,19,15 \\
25,41\end{array}$ \\
\hline $3,15,42$ & 3 & $4,21,38$ \\
\hline $4,21,27$ & 4 & $\begin{array}{l}6,14,7,10,9,16,17,22,23 \\
32,34,35,37\end{array}$ \\
\hline $6,14,43$ & $\mathbf{5}$ & $6,14,43$ \\
\hline $7,10,11,19,8,9,16,17,22,23$ & 6 & 26 \\
$25,18,32,35,41,36,44,54$ & & \\
\hline 26 & 7 & 27 \\
\hline $29,30,31,40,58$ & 8 & $29,30,31,40,58$ \\
\hline $33,37,38,52,59$ & 9 & $33,59,36,44,54$ \\
\hline 46,51 & 10 & $42,43,47,48,45,53$ \\
\hline 49 & 11 & $46,51,50$ \\
\hline 55 & 12 & 49,55 \\
\hline 57 & 13 & 57 \\
\hline $60,64,66$ & 14 & $52,64,66$ \\
\hline 61 & 15 & 57,60 \\
\hline 62 & 16 & 61 \\
\hline 63,67 & 17 & 62 \\
\hline 65 & 18 & 63,67 \\
\hline 68,69 & 19 & 65 \\
\hline 70 & $\mathbf{2 0}$ & 70 \\
\hline
\end{tabular}


Table 6: Cluctering under TD

Table 7: Clustering under $B U$.

\begin{tabular}{|c|c|c|}
\hline Cluster Members & Cluster ID & Cluster Members \\
\hline $\begin{array}{l}1,5,20,31,13,24,28,39,2, \\
12,32,34,41,46,51\end{array}$ & 1 & $1,5,53,39$ \\
\hline $3,4,15,6,14,19,25$ & 2 & $\begin{array}{l}2,12,13,20,24,28,3, \\
11,19,15,25,41\end{array}$ \\
\hline $7,18,9,10,11,17,22,23$ & 3 & $4,21,38$ \\
\hline 8,16 & 4 & $\begin{array}{l}6,14,7,10,9,16,17 \\
22,23,32,34,35,37\end{array}$ \\
\hline 21,27 & 5 & $6,14,43$ \\
\hline $26,40,58,50,38,52$ & 6 & 26 \\
\hline 29,30 & 7 & 27 \\
\hline $33,37,35,44$ & 8 & $29,30,31,40,58$ \\
\hline $36,42,43,48$ & 9 & $33,59,36,44,54$ \\
\hline 45 & 10 & $42,43,47,48,45,53$ \\
\hline $47,56,67,53$ & 11 & $46,51,50$ \\
\hline 49,54 & 12 & 49,55 \\
\hline 55,59 & 13 & 57 \\
\hline 57 & 14 & $52,64,66$ \\
\hline 60,66 & 15 & 57,60 \\
\hline 57 & 16 & 61 \\
\hline 61 & 17 & 62 \\
\hline 63 & 18 & 63,67 \\
\hline 65 & 19 & 65 \\
\hline 70 & 20 & 70 \\
\hline
\end{tabular}

Table 6: Clustering under TD

\begin{tabular}{|l|c|l|}
\hline \multicolumn{1}{|c|}{ Cluster Members } & $\begin{array}{c}\text { Cluster } \\
\text { ID }\end{array}$ & \multicolumn{1}{|c|}{ Cluster Member's } \\
\hline $\begin{array}{l}1,5,20,31,13,24,28,39,2,12,32, \\
34,41,46,51\end{array}$ & $\mathbf{1}$ & $1,5,53,39$ \\
\hline $3,4,15,6,14,19,25$ & $\mathbf{2}$ & $2,12,13,20,24,28,3,11,19,15,25$, \\
\hline $7,18,9,10,11,17,22,23$ & 3 & $4,21,38$ \\
\hline 8,16 & $\mathbf{4}$ & $6,14,7,10,9,16,17,22,23,32,34$, \\
\hline 21,27 & $\mathbf{5}$ & $6,14,43$ \\
\hline $26,40,58,50,38,52$ & 6 & 26 \\
\hline 29,30 & $\mathbf{7}$ & 27 \\
\hline $33,37,35,44$ & 8 & $29,30,31,40,58$ \\
\hline $36,42,43,48$ & 9 & $33,59,36,44,54$ \\
\hline 45 & 10 & $42,43,47,48,45,53$ \\
\hline $47,56,67,53$ & 11 & $46,51,50$ \\
\hline 49,54 & $\mathbf{1 2}$ & 49,55 \\
\hline 55,59 & $\mathbf{1 3}$ & 57 \\
\hline 57 & $\mathbf{1 4}$ & $52,64,66$ \\
\hline 60,66 & $\mathbf{1 5}$ & 57,60 \\
\hline 57 & 16 & 61 \\
\hline 61 & $\mathbf{1 7}$ & 62 \\
\hline 63 & $\mathbf{1 8}$ & 63,67 \\
\hline 65 & $\mathbf{1 9}$ & 65 \\
\hline 70 & $\mathbf{2 0}$ & 70 \\
\hline
\end{tabular}


Table 8: Clustering under WAF.

\begin{tabular}{|l||}
\hline \multicolumn{1}{|c||}{ Cluster Members } \\
\hline $1,5,4,3,15$ \\
\hline $2,24,28,13,20,31,39,51$ \\
\hline $6,17,14,22,23,7,10,11,19,25$ \\
\hline $8,9,16,18,35,12,32,34$ \\
\hline $21,27,38$ \\
\hline $26,50,33,37$ \\
\hline 29,30 \\
\hline $36,43,54,41,42,44$ \\
\hline 40,58 \\
\hline $45,53,47,48$ \\
\hline 46,49 \\
\hline $52,64,66$ \\
\hline 55,59 \\
\hline 56,67 \\
\hline 57 \\
\hline $60,63,68,69$ \\
\hline 61 \\
\hline 62 \\
\hline 65 \\
\hline 70 \\
\hline
\end{tabular}

Table 10: Clustering under PCA.

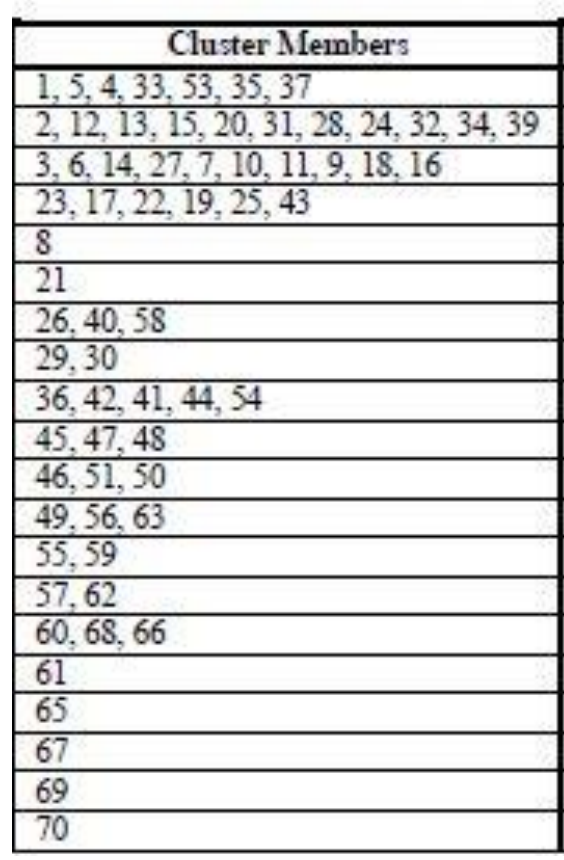

Table 9: Clustering under $B C P$.

\begin{tabular}{|l|l|}
\hline Cluster $\mathbb{D}$ & \multicolumn{1}{|c|}{ Cluster Members } \\
\hline $\mathbf{1}$ & $1,5,39,32,24,41$ \\
\hline $\mathbf{2}$ & $2,12,13,24,28,20,31,26,30$ \\
\hline 3 & $3,33,35,37$ \\
\hline $\mathbf{4}$ & $4,21,27,6,14,11,15,19,25$ \\
\hline $\mathbf{5}$ & $7,8,10,23,9,17,18,22,16,36$ \\
\hline 6 & $38,52,44,54$ \\
\hline $\mathbf{7}$ & $40,58,45,50$ \\
\hline 8 & $42,43,48$ \\
\hline 9 & 46,51 \\
\hline 10 & 47,53 \\
\hline 11 & 49,69 \\
\hline 12 & 55,59 \\
\hline 13 & 56 \\
\hline 14 & 57 \\
\hline 15 & $50,68,62,66,64$ \\
\hline 16 & 61 \\
\hline 17 & 63 \\
\hline 18 & 65 \\
\hline 19 & 67 \\
\hline $\mathbf{2 0}$ & 70 \\
\hline & \\
\hline
\end{tabular}

The degrees to which the clustering of the original dataset (by $C D M$ ) is preserved by each individual dimensionality reduction approach and by the ensemble clustering approach are evaluated using the Rand index [Rand]. The results obtained are shown in Table 12.

Table 11: Final Clustering obtained from the Cluster Ensemble Approach

\begin{tabular}{|c|l|}
\hline Cluster ID & \multicolumn{1}{|c|}{ Cluster Members } \\
\hline $\mathbf{1}$ & $1,5,39,32,34$ \\
\hline $\mathbf{2}$ & $2,13,20,24,28,12,31,29,30$ \\
\hline 3 & $3,15,4,6,14,11,19,25$ \\
\hline $\mathbf{4}$ & $7,10,17,22,23,9,16,8,18$ \\
\hline $\mathbf{5}$ & 21,27 \\
\hline $\mathbf{6}$ & $26,50,40,58$ \\
\hline $\mathbf{7}$ & $33,35,37$ \\
\hline $\mathbf{8}$ & $36,44,54,41,42,43$ \\
\hline $\mathbf{9}$ & 38,52 \\
\hline $\mathbf{1 0}$ & $45,48,47,53$ \\
\hline $\mathbf{1 1}$ & 46,51 \\
\hline $\mathbf{1 2}$ & 49,69 \\
\hline $\mathbf{1 3}$ & 55,59 \\
\hline $\mathbf{1 4}$ & 56,67 \\
\hline $\mathbf{1 5}$ & $60,68,66$ \\
\hline $\mathbf{1 6}$ & $60,68,64,66,62$ \\
\hline $\mathbf{1 7}$ & 61 \\
\hline $\mathbf{1 8}$ & 63 \\
\hline $\mathbf{1 9}$ & 65 \\
\hline $\mathbf{2 0}$ & 70 \\
\hline & \\
\hline
\end{tabular}


Table 12. Agreement between clustering in the original space, each of the DR procedures considered, and ensemble clustering result.

\begin{tabular}{l|lllllllll}
\hline \hline Rand Index & $\mathbf{0 . 9 2 8 4}$ & 0.9275 & 0.9275 & 0.9275 & 0.9043 & 0.9023 & 0.9006 & 0.8928 & 0.8923 \\
\hline \hline $\begin{array}{l}\text { DR } \\
\text { procedure }\end{array}$ & $\begin{array}{l}\text { Ensemble } \\
\text { Clustering }\end{array}$ & DA & BU & BCP & CA & Var & WAF & TD & PCA \\
\hline \hline
\end{tabular}

As it can be seen from this table, the clusters obtained from the ensemble clustering approach have the highest agreement with the clusters obtained in the original dimensions. The clusters obtained from the PCA reduction have the lowest agreement with the clusters obtained in the original dimensions. This behavior of the PCA dimensionality reduction technique with respect to cluster preservation has also been observed in [4].

\section{Conclusion}

This study presents the initial results of the integration of context dependent similarity measures, dimensionality reduction and ensemble clustering approach. The context of two data points, defined with respect to the remaining data points, impacts the evaluation of their similarity. Several dimensionality reduction approaches are considered, each capturing different aspects of the data set. These include seven approaches which reduce the original set of dimensions to a proper subset, as well as the well-known PCA approach in which each dimension in the reduced set is a linear combination of the original dimensions. Experimental results on an artificial data set show that the ensemble clustering agrees the most with the clusters in the original data set. In addition, the clustering in a reduced dimensional space where the dimensions are a proper subset of the original dimensions agrees more with the clustering in the original dimensions set, than that in the set obtained by PCA reduction. These preliminary results warrant further investigation along the lines put forward in this study.

\section{Acknowledgments}

Anca Ralescu's contribution to this work was partially supported by University of Oviedo, University of Osaka.

\section{References}

[1] D. Achlioptas, "Random Matrices in Data Analysis", In Lecture Notes in Computer Science, vol. 3302, In Proceedings of the $8^{\text {th }}$ European Conference on Principles and Practice of Knowledge Discovery in Databases, (2004), pp. 1-7.

[2] E. F. Combarro, E. Montañés, I. Díaz, J. Ranilla and R. Mones, "Introducing a Family of Linear Measures for Feature Selection in Text Categorization”, IEEE Transactions on Knowledge and Data Engineering, vol. 17, no. 9, (2005), pp. 1223-1232.

[3] R. Duda and P. Hart, "Pattern Classification and Scene Analysis", John Wiley, New York, (1973).

[4] X. Z. Fern and C. E. Brodley, "Random Projection for High Dimensional Data Clustering: A Cluster Ensemble Approach", In Proceedings of the Twentieth International Conference on Machine Learning (ICML-2003), Washington DC, (2003), pp. 185-192.

[5] A. D. Gordon, "Classification: Monographs on Applied Probability and Statistics", Chapman and Hall, (1981).

[6] J. A. Hartigan, "Clustering Algorithms", Wiley. MR0405726. ISBN 0-471-35645-X, (1975).

[7] A. Jain and R. Dubes, "Algorithms for Clustering Data", Prentice Hall, New Jersey, (1988).

[8] D. MacKay, "Information Theory, Inference and Learning Algorithms", Cambridge University Press, (2003).

[9] B. Manley, "Multivariate Statistical Models: A Primer", Chapman and Hall, (1986). 
[10] E. Montañés, E. F. Combarro, I. Díaz and J. Ranilla, "Towards Automatic and Optimal Filtering Levels for Feature Selection in Text Categorization”, Lecture Notes in Computer Science, vol. 3646, (2005), pp. 239248.

[11] E. Montañés, J. R. Quevedo and I. Díaz, "A Wrapper Approach with Support Vector Machines for Text Categorization”, Lecture Notes in Computer Science, vol. 2686, (2003), pp. 230-237.

[12] E. Montañés, I. Díaz, J. Ranilla, E. F. Combarro and J. Fernández, "Scoring and Selecting Terms for Text Categorisation", IEEE Intelligent Systems, vol. 20, no. 3, (2005), pp. 40-47.

[13] A. Nsang, "Novel Approaches to Dimensionality Reduction: An Empirical Study", Lambert Academic Publishing, Saarbrücken, Germany, (2011).

[14] A. Nsang and A. Ralescu, "Approaches to Dimensionality Reduction to a Subset of the Original Dimensions", In Proceedings of the Twenty-First Midwest Artificial Intelligence and Cognitive Science Conference (MAICS 2010), (2010), pp. 70-77.

[15] A. Nsang and A. Ralescu, "A Review Of Dimensionality Reduction Methods and their Applications", In Proceedings of the Twentieth Midwest Artificial Intelligence and Cognitive Science Conference, (2009), pp. 118-123.

[16] A. Nsang and A. Ralescu, "More Dimensionality Reduction to a Subset of the Original Attribute Set", In Proceedings of the Twenty-First Midwest Artificial Intelligence and Cognitive Science Conference (MAICS 2010), (2010), pp. 109-116.

[17] M. Rawashdeh and A. Ralescu, "A Pairwise Distance View of Cluster Validity", Advances on Computational Intelligence, Springer, (2012), pp. 561-570.

[18] R. S'winiarski, K. Cios and W. Pedrycz, "Data Mining Methods for Knowledge Discovery", Kluwer Academic, ISBN 0-7923-8252-8, (1998).

[19] W. D. Tembe, "Pattern Extraction Using Context Dependent Measure of Divergence and its Validation", MSc Thesis, University of Cincinnati, (2001).

[20] W. Tembe and A. Ralescu, "Fuzzy algorithm for contextual character recognition", In Proceedings of the 2004 IEEE International Conference on Fuzzy Systems, vol. 3, (2004), pp. 1733-1738.

[21] W. D. Tembe, "Proximity Metrics for Contextual Pattern Recognition", PhD Thesis, University of Cincinnati, (2004). 